\title{
Neue Herausforderungen im stationären Sektor ab 2012
}

\author{
Tilman Slembeck ${ }^{a}$, \\ Roswitha Scheidweiler ${ }^{b}$ \\ a Prof. Dr. oec., Professor \\ für Volkswirtschaftslehre, \\ Zürcher Hochschule für \\ Angewandte Wissenschaften \\ und Universität St.Gallen \\ b Seminarleitung, RS Medical \\ Consult $\mathrm{GmbH}$
}

Die kommenden Jahre bringen dem stationären Sektor der Schweiz einen massiven Umbruch. Unter dem Stichwort «Neue Spitalfinanzierung» ist auf politischer Ebene eine Reihe von Neuerungen in Gang gesetzt worden, welche die Spitäler, aber auch die vor- und nachgelagerten Institutionen vor neue Herausforderungen stellen werden. Neben der per 2012 geplanten Einführung von stationären Fallpauschalen (DRGs) betrifft dies etwa die neue Spitalplanung der Kantone, die freie Spitalwahl ausserhalb des Wohnkantons sowie die Offenlegung von Daten und Qualitätsindikatoren. Damit werden neue Rahmenbedingungen geschaffen, die nicht nur einheitlichere Finanzierungsregeln, sondern auch eine höhere Transparenz schaffen und zu einer Sicherung der Qualität und des Wettbewerbs im Gesundheitswesen beitragen sollen. Wie die Rahmenbedingungen im Einzelnen ausgestaltet sein werden und ob die angestrebten Wirkungen eintreten werden, ist bislang offen. Sicher ist hingegen, dass sich alle Leistungserbringer, vor allem aber die Spitäler, neu orientieren, strategisch ausrichten und reorganisieren müssen.

Die zentrale Frage lautet deshalb: Was kommt auf die Leistungserbringer und die Spitäler im Speziellen zu und wie können sie auf die Herausforderungen reagieren? Speziell interessant sind folgende Aspekte:

- Unter welchen neuen Rahmenbedingungen werden Kantone und Spitäler in Zukunft agieren?

- Wie wirken sich die neuen Rahmenbedingungen auf die Spitallandschaft insgesamt aus?

- Wie können sich einzelne Spitäler im Wettbewerb strategisch positionieren und organisatorisch vorbereiten?

Ziel des Symposiums ist es, diese Fragen zu klären, Chancen und Probleme rund um das Thema «Neue Spitalfinanzierung» auszuloten und Handlungsoptionen zu diskutieren.

\section{Programm Symposium «Neue Spitalfinanzierung», 15. Januar 2010, Marriott Hotel, Zürich}

Ab 8.30 Uhr

$9.00 \mathrm{Uhr}$

9.05-09.15 Uhr

9.15-9.45 Uhr

9.45-10.15 Uhr

10.35-11.05 Uhr Lic. rer. pol. Stefan Kaufmann, Direktor santésuisse: Die «neue Spitalfinanzierung» aus Sicht der Versicherer

11.05-11.35 Uhr Dr. med. Pierre-François Cuénoud, Mitglied Zentralvorstand FMH: Die «neue Spitalfinanzierung» aus Sicht der Medizin

11.35-12.00 Uhr Plenumsdiskussion 1 (mit den Referenten des Vormittags)

12.00-13.00 Uhr Mittagessen

13.45-14.15 Uhr Prof. Dr. Johannes Rüegg-Stürm, Dir. Institut für Betriebswirtschaft der Universität St. Gallen: Prozessoptimierung als konstruktive Praxis einer integrativen Spitalentwicklung

14.15-14.45 Uhr Dr. Arnold Bachmann, Vizepräsident H+: Neue Rahmenbedingungen für die Spitäler: Mehr als nur DRGs?

15.15-15.45 Uhr Joseph Rohrer,Vorsitzender der Geschäftsführung, Universitätsklinikum Giessen und Marburg GmbH: Transparenz, Qualität und Wettbewerb als strategische Herausforderung

15.45-16.45 Uhr Plenumsdiskussion 2 (mit den Referenten des Nachmittags)

16.45 Uhr Schlusswort - Gedankenaustausch unter den Teilnehmenden beim Apéro

Weitere Informationen zum Symposium und Online-Anmeldung unter www.rsmedicalconsult.com 